\title{
REPLICATION IMAGE DETECTION USING CONVOLUTIONAL NEURAL NETWORK
}

DOI : 10.36909/jer.ICIPPSD.15499

\author{
K. Kalaiselvi", S. Saranya ${ }^{* *}$, K. Deepa Thilak* ${ }^{*}$ K. Kumaresan ${ }^{* * *}$ \\ *Department of Computer Science and Engineering, SRM Institute of Science \& Technology, \\ Kancheepuram, Tamil Nadu, India \\ ** Department of Electronics and Communication Engineering, Easwari Engineering College, \\ Ramapuram, Chennai, Tamil Nadu, India \\ *** Department of Computer Science and Engineering, KSR College of Engineering, \\ Tiruchengode, Tamil Nadu, India \\ *Email: mkkalai1981@gmail.com ; Corresponding Author.
}

\begin{abstract}
The ease of access to image data has led to overuse of repeated images at various instances which leads to increases duplication and redundancy in many industries. Advanced editing techniques which are available very easily, encourages original copyrights images to be misused. This results in lack of originality in data generated at every level. Common solutions include allowing manual selections of duplicate images or compares images pixel by pixel. The conventional method is to use 3 branch Siamese Convolution model to detect duplication of medical images. We propose to develop a model to detect duplication in everyday images by training a Siamese Convolutional Neural Network and try to achieve greater accuracy than previously developed solutions. Using Grad-Cam network inspection we propose to inspect the decisions taken by the CNN upon detecting duplication in images.
\end{abstract}

Keywords: Neural Network, Data Processing, Duplication, Grad-CAM.

\section{INTRODUCTION}

We are addicted to electronic gadgets in many ways. Some play games, some change the UI, booting programs, some learns, some hacks and some watch videos, and mostly everyone is using smartphones for texting and for calling in means of communication. This is the generation of internet through which we share and get access to enormous data in social media platforms daily. There are many social media platforms through which we can share our talents and can explore new ideas and various visions. One can share images, quotes, videos and many more things. About 2.5 quintillion bytes of data is generated every minute. Research states that about $28.7 \%$ of data images generated is duplicated of previously generated data most of which is shared on social media platforms. A duplicate detection model will 
enable us in preventing duplicate data generations, thus not wasting internet resources and unwanted consumption of spaces in mobile. Duplication of images has its effects in machine learning and deep learning industries also. Data processing is a key step in major data science and deep learning projects where each data provides valuable information and contributes greatly to the accuracy of the model developed. Data sets of images can be easily downloaded from many sources as required. While finding the right format of the image dataset is a challenge, making sure that there are no duplications among the images is another yet important challenge. Duplication in these datasets, would negatively impact the model. It leads to the common problem of overfitting in models, where the model achieves a high accuracy during training and learns with full accuracy but performs poorly on test data. In these cases, it is said that the model over fits the prediction curve.

Lack of duplication identification step in the preprocessing phase, leads to unnecessary suspects and changes in the model when the model overfits the data. The first step that would be taken in resolving the issue would be to increase the scope of the dataset. Next, the model is modified accordingly. Depending on the error rate, the test and train data split range is changed. Many times, the duplication issue of the image dataset goes unidentified or unnoticed and causes problems in the accuracy. By applying a duplication detection algorithm on the dataset that is to be used as input to another model, duplicates can be identified and removed thus making pre-processing phase on the dataset more efficient and profitable.

The current model has is not completely accurate as it faces difficulties while finding duplicates in certain type of image sets. The model however makes significant errors in duplication detection when contrast and saturation in the image is changed. The existing models are trained to identify duplicates in images if they are edited or cropped. However, changes in the color, depth and lightness is difficult to identify. The scope of the dataset is limited to a particular field and not to day-to-day objects. The proposed model will be very helpful in creating pre-processed data set, where the existence of duplicate data is a huge loss to data scientists and machine learning. The proposed system consists of Siamese Convolutional Neural Network with three layers and Grad Cam used for extracting the information of the resulted duplicate images and to influence the decisions of $\mathrm{CNN}$. The proposed model

identifies the duplicates and indicates how it arrived at the decision with the help of the Grad-CAM. It identifies images that have even been slightly modified to escape duplication but hey are both the same images (Q. Li. and P. Wu., 2020). The model also knows to determine if the given dataset of images do not have any duplicates in them. It can also determine whether two images are different that is, non duplicate. Depending on the dataset's scope and size, the model's accuracy varies, and duplication can be identified better with increase in efficiency and experience for the model. 


\section{LITERATURE SURVEY}

(Jia Song et.al.,2019, C. Wang.,2020) CNNs have accomplished striking accomplishment in PC vision. As of late, many specialists have examined distant detecting picture order utilizing CNNs, and CNNs can be applied to acknowledge fast, affordable, and exact investigation and highlight extraction from distant detecting information. This paper intends to give a review of the present status of-theworkmanship use of CNN-based profound learning in far off detecting picture order. We first momentarily present the standards and qualities of CNNs. We at that point review advancements and primary enhancements for CNN models that make CNNs more reasonable for far off detecting picture characterization, accessible datasets for far off detecting picture order, and information increase procedures. At that point, three run of the mill CNN application cases in distant detecting picture grouping: scene characterization, object location and article division are introduced. (Marco Mora et. al.,2019). Agriculture has usually been a vital monetary and social quarter for humans. Fruit manufacturing is especially crucial, with an extraordinary call for from all families. Therefore, the usage of present-day generation is of essential significance for the Agri-meals zone. Currently synthetic intelligence is one very essential technological tool considerably applied in modern-day society. Particularly, Deep Learning (DL) has several applications because of its capability to study sturdy representations from images. Convolutional Neural Networks $(\mathrm{CNN})$ is the primary DL structure for picture class. Based at the first-rate interest that CNNs have had within the closing years, we gift a evaluation of the usage of $\mathrm{CNN}$ achieved to certainly one of a kind automated processing obligations of fruit pix: category, amazing control, and detection. We have a look at that in the last years (2019-2020), using $\mathrm{CNN}$ for fruit reputation has extensively extended obtaining high-quality consequences, both via the usage of new models or with pre-educated networks for switch learning. It is well worth noting that distinctive types of snap shots are utilized in datasets consistent with the assignment finished. Besides, this article gives the fundamentals, tools, and examples of the use of CNNs for fruit sorting and pleasant manage. (Irfan Uddin et.al.,2020) Coronavirus, a dangerous illness that began in Wuhan, China, has brought about a worldwide episode. Patients contaminated with the causative infection SARS-CoV-2 are put in isolate, so the infection doesn't spread. The clinical local area has not found any immunization that can be quickly utilized on patients contaminated with SARS-CoV-2. The lone strategy found so far to shield individuals from this infection is staying away from others, wearing covers and gloves, just as consistently washing and purifying hands. Government and law requirement organizations are engaged with restricting the development of individuals in various urban areas, to control the spread and screen 
individuals following the rules of the CDC. (Cohen and D. Normile, 2020) However, it isn't workable for the public authority to screen all spots, for example, shopping centers, medical clinics, government workplaces, and banks, and direct individuals to follow the security rules. In this paper, a novel method is built up that can direct individuals to secure themselves from somebody who has high openness to the infection or has manifestations of COVID-19, for example, having fever and hacking. Diverse profound Convolutional Neural Networks ( $\mathrm{CNN})$ models are actualized to test the proposed strategy. The proposed canny observing framework can be utilized as an integral instrument to be introduced at better places and consequently screen individuals receiving the wellbeing rules. With these prudent estimations, people will be capable to win this battle against COVID-19.

Ciconnet (2019) has established in their paper that the Siamese Convolutional network when trained on a dataset has the potential to detect image manipulation and duplication by training the network on medical images. Their model has identified duplications to a significant extent and has made some incorrect predictions in images whose contrast and histogram have been changed. Morro (2019) has established that the convolutional network can be used to identify differences between near-duplicate and non-near duplicate images. The model is modified into a binary classification problem and image pairs are differentiated. The accuracy achieved by the model establishes that near-duplicate image identification is possible to a significant level. Yi Zhang (2018) has established that neural network models can learn the similarities between images. A pair of images are jointly processed and using a similarity function the images are identified as either a near-duplicate or a different image.

Dong (2018) has established that the false positive rates in near duplicate detection models can be reduced to a very insignificant value by using entropy-based filtering technique that eliminates ambiguous features and thus achieving high confidence near-duplicate image identification. Foo (2007) has established that the importance of image redundancy in web search queries where manipulation of images is different an identification of redundant images is essential. The model's performance and accuracy are compared to those of other matching algorithms. It establishes that automatic near-duplicate identification in images can be used in web search. Qi (2016) has established that the Siamese CNN can be used to segregate pairs of images based on the output vector after processing by collecting the relevant ones and discarding the irrelevant ones. It has explained the identification, similarity, and differences in Siamese network in a pair of images. Dhlamini (2019) has established the performance of Siamese networks in comparing and identifying the author from handwriting identification. It explains the comparing and successful identification as the output of the model. Huang (2020) has established that trained Siamese CNN can be used to identify the similarity in images and hence can identify the images as in the case of object detection mentioned in the model. 


\section{PROPOSED MODEL}

Traditionally, which include colour to black and white photographs changed into achieved via hand with human strive due to the fact it's far this type of hard task. Deep studying can use the items and their context within the photo to colour the picture, much like a human operator could possibly technique the trouble. This is performed through using high first-class and big convolution neural networks and supervised layers that recreate the photograph with the addition of colour. There are packages to be had that let you simply take a photo of a textual content written in some remote places language and translate it with the intention to be look at in a language that you could understand. That is a deep getting to know community. It can mechanically translate given terms, phrase, or sentences in a single language into every different language as you will assume. It makes use of convolution neural networks to discover pictures that have letters. Once recognized, they can have become text, translated, and recreates the photograph with the translated text. This is regularly known as on the spot seen translation.

Similarly, text translation can be finished without any pre-processing of the collection, permitting the algorithm to research the dependencies between words and their mapping to a brand-new language. Machine studying provided a exclusive method to solving laptop imaginative and prescient troubles. With device getting to know, developers no longer had to manually code every unmarried rule into their imaginative and prescient applications. Instead, they programmed smaller packages that would discover styles in photographs. They then used a statistical gaining knowledge of set of rules inclusive of linear regression, logistic regression, selection trees or assist vector machines (SVM) to stumble on styles and classify photographs and stumble upon gadgets in them.

Traditionally, including colour to black and white images changed into achieved by hand with human attempt because it is any such tough mission. Deep learning can use the objects and their context inside the image to colour the photo, much like a human operator would possibly technique the hassle. This is done by the usage of high fine and huge convolution neural networks and supervised layers that recreate the picture with the addition of color. There are packages available that allow you to just take a photo of a textual content written in a few overseas languages and translate it so as to be examine in a language that you may understand. That is a deep gaining knowledge of network. It can robotically translate given phrases, phrase, or sentences in a single language into every other language as you will assume. It makes use of convolution neural networks to discover pictures that have letters. Once recognized, they can be 
becoming textual content, translated, and recreates the picture with the translated textual content. This is regularly called immediate visible translation.

\section{Proposed CNN model Algorithm}

Step 1: Starts with an enter picture

Step 2: Applies many special filters to it to create a feature map

Step 3: Applies a ReLU function to increase non-linearity

Step 4: Applies a pooling layer to every characteristic map

Step 5: Flattens the pooled pics into one long vector.

Step 6: Inputs the vector into a fully related artificial neural network

Step 7: Inputs the vector into a totally connected synthetic neural community.

Step 8: Processes the features via the community. The final absolutely connected layer and provides the "balloting" of the instructions that we're after.

Step 9: Trains through forward propagation and backpropagation for many, many epochs.

Step 10: This repeat till we have a well-described neural network with trained and feature detectors.

Computer imaginative and prescient additionally performs an essential role in facial reputation packages, the era that enables computer systems to fit photographs of humans' faces to their identities. Computer imaginative and prescient algorithms locate facial functions in photos and examine them with databases of face profiles. Consumer gadgets use facial popularity to authenticate the identities in their owners. Social media apps use facial reputation to detect and tag customers. Law enforcement businesses additionally rely upon facial popularity generation to discover criminals in video feeds.

The model proposed in this paper is a Siamese CNN model which is used to simultaneously process a pair of images. A pair of images are sent as input to the model which then classifies the image as duplicate or non-duplicate. The model is trained on images that are duplicates and images that are not duplicates of an anchor image. The Airbnb Dataset of hotel rooms is used to train this model. Image editing works such as editing, cropping, contrast alteration, saturation alteration is made on the anchor images to train the model on various ways of duplication of images. An initiative is taken to apply GradCAM technique to try to visualize the model's decision. The results from Grad-CAM can be used to improve the results and to better analyse the model. 
Siamese convolutional neural networks are mainly used in models that require similarity detections and comparison of images and their features. There are two conv nets and each of them takes an input and processes it based on its instructions. A conv net is a set of multiple layers performing convolution, pooling, and filtering with he given input and produces an individual output. The other conv net also takes a similar type of input as that the comparison in their outputs is possible and valid. Duplication of images is a common problem that exists in all fields that work with digital images. Using watermark has been a common solution but advanced editing tools have made replicating copyright images easier.

Identifying similarity and duplication in images help research scholars to make sure there is no data duplication in areas such as data set, data repository and sample data presented in the review. Implementation is the process of making the proposed model execute successfully. It comprises of the successful achievement of a few milestones in installation and setting up of environment. The implementation of our Siamese CNN model involves two phases. The initial implementation phase is the installation of Jupiter Notebook and TensorFlow along with the required packages. The next step in implementation is the elaborate coding that implements the proposed model by executing the code with the input data set that is divided into the train and test data for the different phases respectively.

The first module is about developing a convolutional neural network model that will detect replicates in images with the help of contrastive loss. The Convolutional Neural Network In this module, the concentration is mainly on the performance and accuracy of the convolutional neural network model. The performance is monitored based on both the execution time and CPU utilization. The accuracy is measured on four different fronts namely, true positive, true negative, false positive and false negative. The output from this module, is used as input for the next module to validate the decisions made in/ this module.

The second module is the implementation of gradient-weighted class activation mapping. Inclination weighted Class Activation Mapping (Grad-CAM) utilizes the slopes of any objective idea streaming into the absolute last convolutional layer to supply a harsh restriction map featuring the significant districts in the photograph for anticipating the thought. We totally take the last convolutional highlight map after which we gauge each divert in that include with the angle of the tastefulness with perceive to the channel. It is just nothing anyway how seriously the information picture turns on remarkable channels by utilizing how imperative each channel is concerning the heavenliness. This module describes the decisions taken by the CNN model in the previous module. It highlights the features in the image samples that lead the CNN to decide on its level of similarity to the pairing image. The proposed model can identify images that are duplicates of one another, by identifying key features in them that are similar or have been slightly modified. Emphasis is laid on improving the accuracy of the model from previously existing 
systems, such as by enabling duplicate identification in images whose contrast and saturation and other filter effects have been added or removed accordingly. In existing models, such type of identification is found to be difficult and hence the proposed model focuses on rectifying the issue in duplicate identifying models and providing a better model. The model implementation has two phases, the CNN building and training and the Grad-CAM implementation phase.

\section{PROCEDURE-PROPOSED CNN MODEL PROCESSING AN IMAGE}

- A regular CNN design may look something like this

- Input - >Convolution - >ReLU - >COnvolution - >ReLU - >Pooling - > ReLU - > Convolution - > ReLU - >Pooling - >Fully Connected Tangle anymore CNN convolves (does not tangles...) learned abilities with input data and utilizations 2D convolutional layers.

- This way that this sort of local area is ideal for preparing 2D pictures. Contrasted with various picture order calculations, CNNs undoubtedly, utilize almost no pre-handling. This technique that they could get familiar with the channels that ought to be hand-made in different calculations.

- CNNs can be utilized in huge loads of bundles from picture and video acknowledgment, photo order, and recommender designs to home grown language handling and logical photograph examination.

- CNNs have an enter layer, and yield layer, and covered up layers. The secret layers for the most part incorporate convolutional layers, ReLU layers, pooling layers, and totally related layers. Convolutional layers apply a convolution activity to the information. This gives the data to the following layer. Pooling joins the yield of bunches of neurons into a solitary neuron in the organization.

The main objective of this project is to implement a reliable and scalable image duplication detected system. Improving the model's accuracy is also an important goal of the model. It aims on identifying and solving the problems and weaknesses which the previous models had, such as unable to detect duplicates when lightness is modified, difficult to detect replicates when the image is edited heavily.

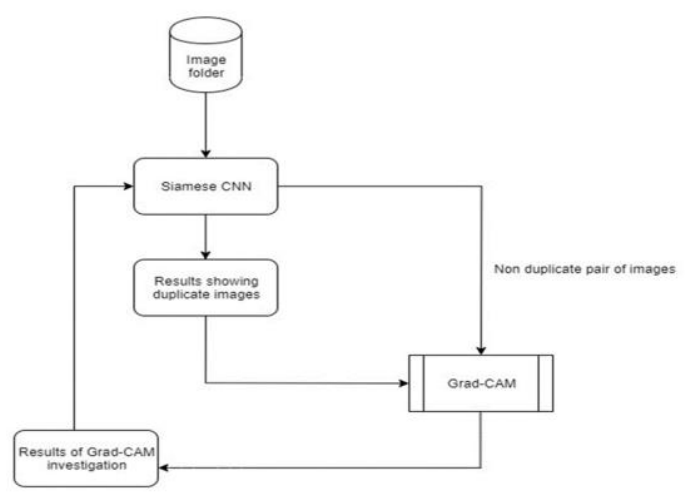

Figure 1 Architecture of the proposed model 


\section{RESULTSAND DISCUSSION}

The Siamese Convolutional neural network model has produced the following results in classifying images

as duplicates and non-duplicates.

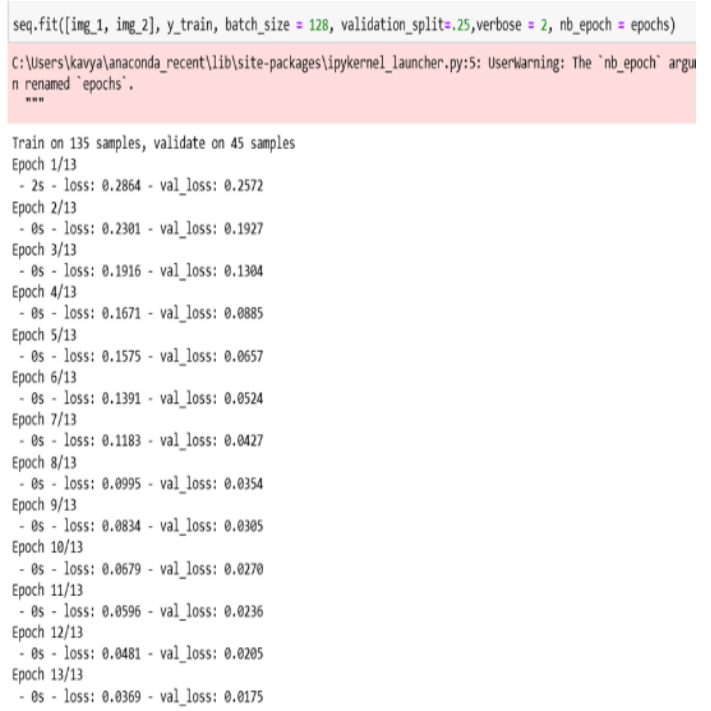

\section{Figure 2 Screenshot of the training results of the model}

Figures 3 to 9 denote cases where the model has rightly identified duplicate pairs of images. Various kinds of image manipulation are done on the original image before passing it as input.
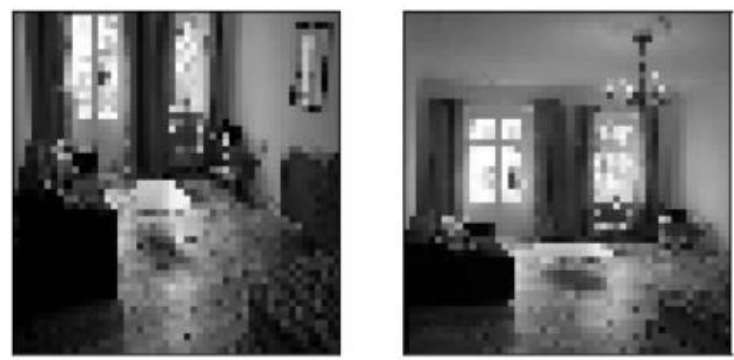

duplicate

Figure 3 Pair of same images where the angle is different 

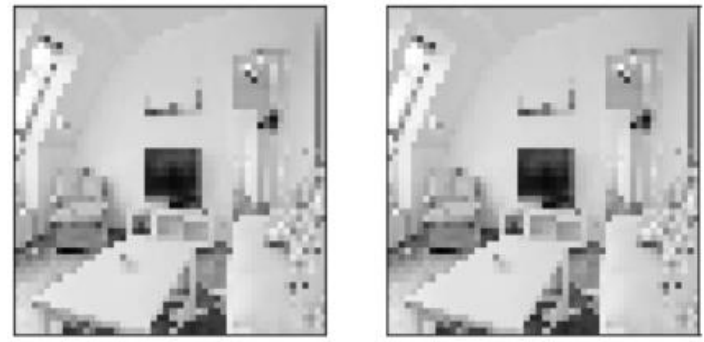

duplicate

Figure 4 Illustration of pixelated picture duplicates of a similar picture
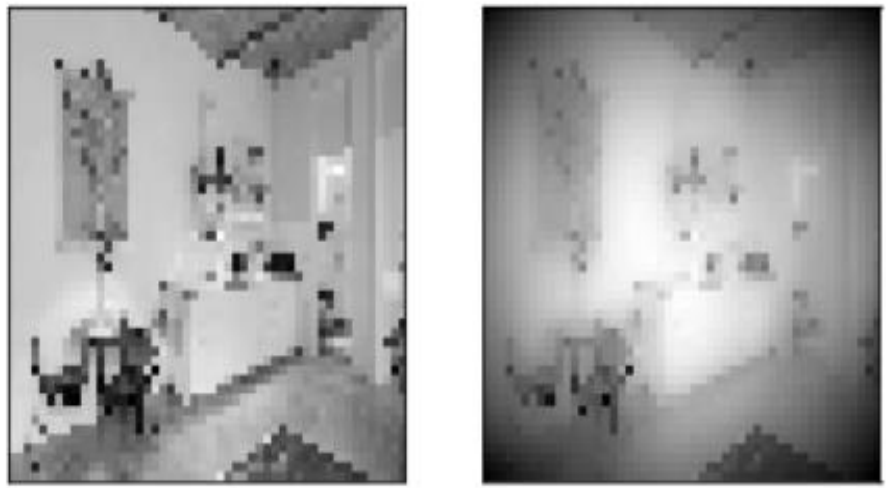

duplicate

Figure 5 Illustration of a pair of same images where one has been edited
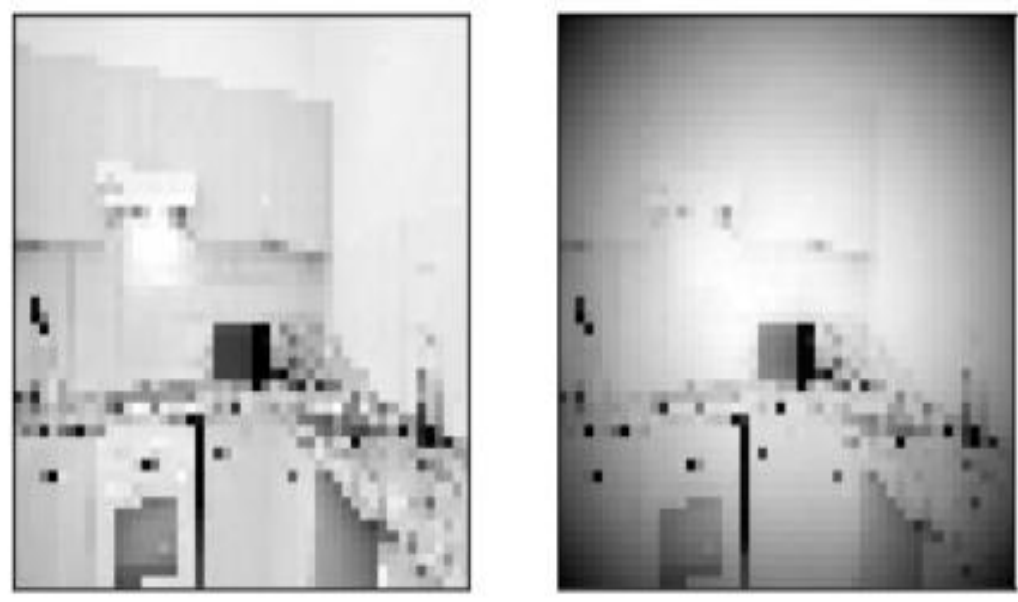

duplicate

Figure 6 Illustration of a couple of same pictures where the angle and contrast are changed 

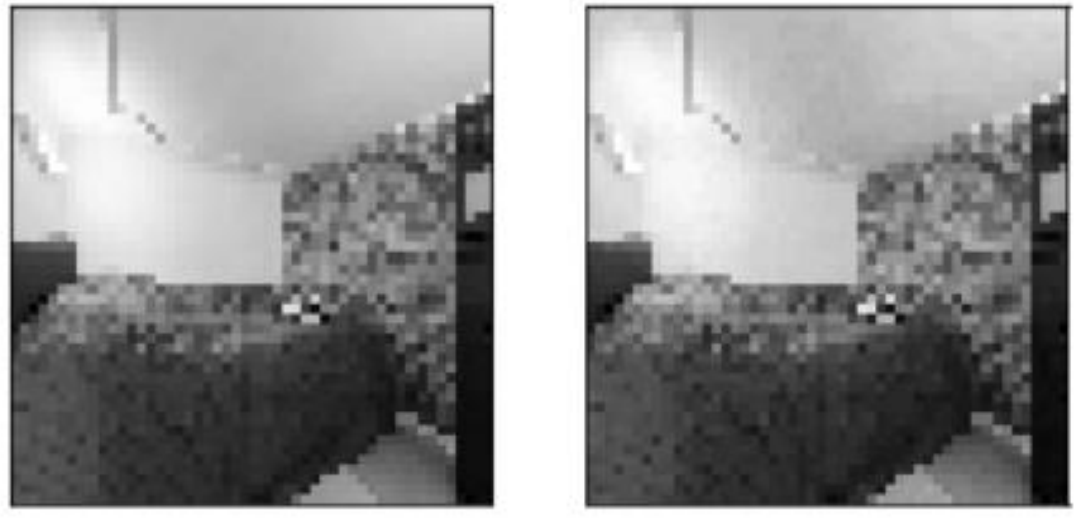

duplicate

Figure 7 Example of a pair of same images with altered Saturation
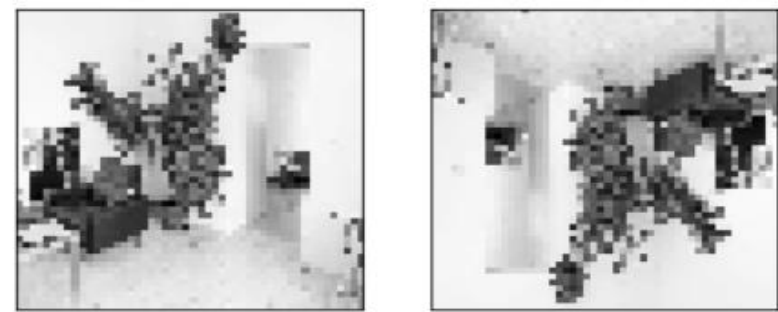

duplicate

Figure 8 Illustration of a pair of same images where the rotation is changed
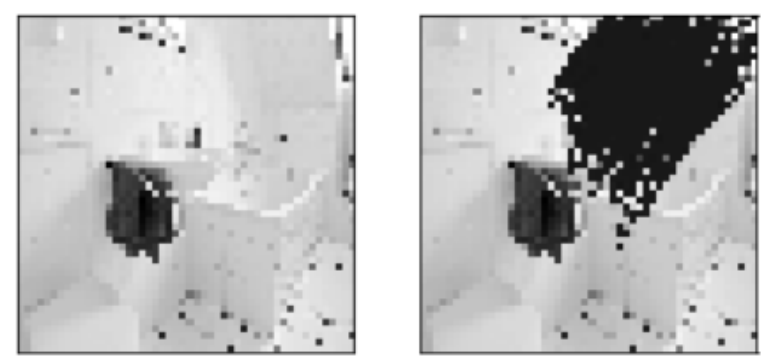

duplicate

Figure 9 Illustration of a couple of same pictures where one is heavily edited

Figure 10 and 111shows the cases where the model has identified a given input pair of images as nonduplicate images. 



non duplicate

Figure 10 Example of a pair of different images with different angles
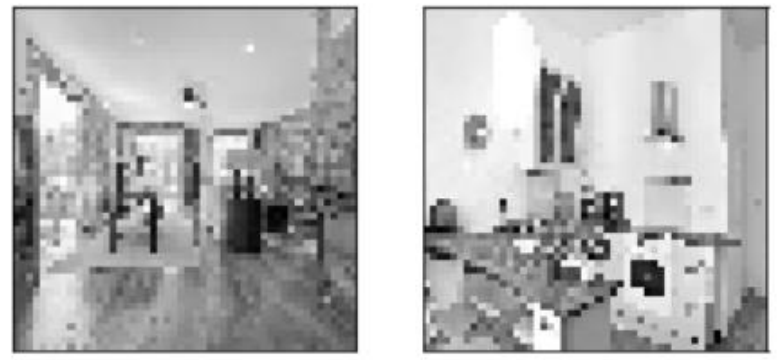

non duplicate

Figure 11 Example of a pair of different images with different objects in it.

\section{CONCLUSION}

The simple entry to picture information has prompted abuse of rehashed pictures at different occurrences which prompts builds duplication and excess in numerous businesses. Progressed altering procedures which are accessible effectively, urges unique copyrights pictures to be abused. This outcomes in absence of inventiveness in information produced at each level. Basic arrangements incorporate permitting manual choices of copy pictures or looks at pictures' pixel by pixel. The customary technique is to utilize 3 branch Siamese Convolution model to distinguish duplication of clinical pictures. We propose to build up a model to recognize duplication in regular pictures via preparing a Siamese Convolutional Neural Network and attempt to accomplish more prominent precision than recently created arrangements. Utilizing Grad-Cam network examination and propose to assess the choices taken by the $\mathrm{CNN}$ after recognizing duplication in pictures

\section{REFERENCES}

C. Wang, P. W. Horby, F. G. Hayden, and G. F. Gao, 2020 "A novel coronavirus outbreak of global health concern," The Lancet, vol. 395, no. 10223, pp. 470-473.

Cohen and D. Normile, 2020. "New SARS-like virus in China triggers alarm," Science, vol. 367, no. 6475, pp. 234-235, 2020. 
Connor Shorten and Taghi M. Khoshgoftaa, Shorten and Khoshgoftaar J Big Data,2019 “A survey on Image Data Augmentation for Deep Learning,"

Jia Song, Shaohua Gaob, Yunqiang Zhua, and Chenyan Mab, 2019, A survey of remote sensing image classification based on CNNs, published by Taylor \& Francis Group and Science Press on behalf of the International Society for Digital Earth, supported by the CASEarth Strategic Priority Research Program.

Jun Jie Foo, Justin Zobel, Ranjan Sinha, and S. M. M. Tahaghoghi. 2007. Detection of nearduplicate images for web search. In Proceedings of the 6th ACM international conference on Image and video retrieval (CIVR '07). Association for Computing Machinery, New York, NY, USA, 557-564. DOI: https://doi.org/10.1145/1282280.1282360

L. Huang and Y. Chen, "Dual-Path Siamese CNN for Hyperspectral Image Classification with Limited Training Samples," in IEEE Geoscience and Remote Sensing Letters, doi: 10.1109/LGRS.2020.2979604.

Lia Morra, Fabrizio Lamberti, 2019, Benchmarking unsupervised near-duplicate image detection, Expert Systems with Applications, Volume 135, Pages 313-326, ISSN 0957-4174, https://doi.org/10.1016/j.eswa.05.002.

M. Ciccone and H. Elliott and D. L. Richmond and D. Weinstock and M. Walsh, year 2019. Image Forensics: Detecting duplication of scientific images with manipulation-invariant image similarity

M. Irfan Uddin, 1 Syed Atif Ali Shah, and Mahmoud Ahmad Al-Khasawneh, 2020, A Novel Deep Convolutional Neural Network Model to Monitor People following Guidelines to Avoid COVID$19, "$

Marco Mora, Ruber Hernández-García Ricardo J. Barrientos, Claudio Fredes and Andres Valenzuela, 2019, A Review of Convolutional Neural Network Applied to Fruit Image Processing José Naranjo-Torres.

N. Dlamini and T. L. van Zyl, "Author Identification from Handwritten Characters using Siamese CNN," 2019 International Multidisciplinary Information Technology and Engineering Conference (IMITEC), Vanderbijl park, South Africa, 2019, pp. 1-6, doi: 10.1109/IMITEC45504.2019.9015897.

Q. Li, X. Guan, P. Wu., 2020., "Early transmission dynamics in Wuhan, China, of novel coronavirusinfected pneumonia,” New England Journal of Medicine, vol. 382, no. 13, pp. 1199-1207

Wei Dong, Zhe Wang, Moses Charikar, and Kai Li. 2012. High-confidence near-duplicate image 
detection. In Proceedings of the 2nd ACM International Conference on Multimedia Retrieval (ICMR '12). Association for Computing Machinery, New York, NY, USA, Article 1, 1-8. DOI: https://doi.org/10.1145/2324796.2324798

Y. Qi, Y. Song, H. Zhang, and J. Liu, "Sketch-based image retrieval via Siamese convolutional neural network," 2016 IEEE International Conference on Image Processing (ICIP), Phoenix, AZ, 2016, pp. 2460-2464, doi: 10.1109/ICIP.2016.7532801.

Zhang, Y., Zhang, Y., Sun, J., Li, H., \& Zhu, Y. (2018). Learning near duplicate image pairs using convolutional neural networks. International Journal of Performability Engineering, 14(1), 168 177. 